\title{
Pathways to Brain Aging and Their Modifiers: Free-Radical-Induced Energetic and Neural Decline in Senescence (FRIENDS) Model - A Mini-Review
}

\author{
Naftali Raz ${ }^{a, b, d}$ Ana M. Daughertyc \\ ${ }^{\mathrm{a}}$ Institute of Gerontology and ${ }^{\mathrm{b}}$ Department of Psychology, Wayne State University, Detroit, MI, and \\ 'Beckman Institute for Advanced Science and Technology, University of Illinois at Urbana-Champaign, \\ Urbana, IL, USA; ' Max Planck Institute for Human Development, Berlin, Germany
}

\section{Keywords}

Mitochondria $\cdot$ Reactive oxygen species - Magnetic resonance spectroscopy · Magnetic resonance imaging · Longitudinal studies · Brain energy metabolism · White matter · Myelin · Neuropil

\begin{abstract}
In this mini-review, we survey the extant literature on brain aging, with the emphasis on longitudinal studies of neuroanatomy, including regional brain volumes and white matter microstructure. We assess the impact of vascular, metabolic, and inflammatory risk factors on the trajectories of change in regional brain volumes and white matter properties, as well as the relationships between neuroanatomical and physiological changes and their influence on cognitive performance. We examine these findings in the context of current biological theories of aging and propose the means of integrating noninvasive measures - spectroscopic indices of brain energy metabolism and regional iron deposits - as valuable proxies for elucidating the basic neurobiology of human brain aging. In a brief summary of the recent findings pertaining to age-related changes in the brain structure and their impact on cognition, we discuss the role of vascular,
\end{abstract}

\section{KARGER}

(C) 2017 S. Karger AG, Basel

E-Mail karger@karger.com

www.karger.com/ger metabolic, and inflammatory risk factors in shaping the trajectories of change. Drawing on the extant biological theories of aging and mindful of the brain's role as a disproportionately voracious energy consumer in mammals, we emphasize the importance of the fundamental bioenergetic mechanisms as drivers of age-related changes in brain structure and function. We sketch out a model that builds on the conceptualization of aging as an expression of cumulative cellular damage inflicted by reactive oxygen species and ensuing declines in energy metabolism. We outline the ways and means of adapting this model, Free-Radical-Induced Energetic and Neural Decline in Senescence (FRIENDS), to human aging and testing it within the constraints of noninvasive neuroimaging.

(c) 2017 S. Karger AG, Basel

Aging is a universal phenomenon that affects virtually all systems and organs. The brain, however, has attracted the attention of gerontologists because of the increased incidence of dementia, for which advanced age is the main risk factor. Understanding age-related neurodegenerative disorders that are associated with catastrophic cognitive declines, and eventually mitigating their most

Naftali Raz

Institute of Gerontology, Wayne State University

87 East Ferry St.

Detroit, MI 48202 (USA)

E-Mailnraz@wayne.edu 
devastating consequences, necessitates understanding alterations to the brain's structure, metabolism, and physiological characteristics that accompany typical and successful aging, which present milder declines that nonetheless negatively affect the daily functioning of millions [1]. This is a daunting task, and in the absence of a clear evolutionary program for aging [2], numerous theories have been advanced to explain the molecular, cellular, or neuroanatomical aspects of aging, including the free-radical hypothesis [3], vascular dysfunction theory [4], and a model emphasizing the critical role of myelin maintenance failure [5].

In this review, we will sketch out a model - Free-Radical-Induced Energetic and Neural Decline in Senescence (FRIENDS) - aimed at bridging various levels and aspects of brain aging and propose strategies for testing its predictions.

\section{Brain Aging: Postmortem Studies}

Postmortem studies in humans and other primates reveal a plethora of age-related brain differences. Advanced age at death is associated with a reduction in gross brain volume and weight; shrinkage, dysmorphology, and selective attrition of neurons; loss of cortical and subcortical myelin; impediment to neurogenesis and synaptogenesis; gliosis; and a shift in the equilibria of minerals and metals [1-8]. The postmortem methodology, however, has one major flaw: the impossibility of elucidating the temporal dynamics of change and the individual differences therein - hence the significant advantage of noninvasive neuroimaging.

\section{Brain Aging: In vivo Evidence}

The extant body of cross-sectional neuroimaging studies is impressive, and they indeed opened a window into age differences in gross brain structure. The cross-sectional in vivo findings agree with the postmortem records, as they document age-related differences in almost all brain regions, including smaller total and regional volumes, a thinner cortex, and larger CSF-filled cavities in persons of advanced age compared to their younger counterparts [1]. The magnitude of the age differences varies across brain structures: greater negative effects of age are observed in the prefrontal cortex, cerebellum, and neostriatum as compared to the primary sensory cortices [1].
The major drawback of cross-sectional designs is the inability to capture an essentially dynamic phenomenon like aging in a single snapshot [9]. The data structure generated by cross-sectional studies does not conform to the ergodicity assumption and thus cannot represent individual time-dependent change [10]. Moreover, cross-sectional mediation models produce biased estimates of effects attributed to the mediators [11]. In contrast to crosssectional studies, longitudinal investigations can estimate the true rate of change and individual variability therein $[9,12]$. They are, however, not without significant limitations: participant attrition, the small number of assessment occasions, and relatively short time windows for assessment. Moreover, many rely on age-heterogeneous samples and thus confound cross-sectional age differences with change. With these constraints in mind, throughout this review we will limit our discussion to the extant longitudinal studies, while calling upon cross-sectionally sourced findings only in cases when longitudinal data are unavailable.

Longitudinal studies of cortical and subcortical volumes of the human brain reveal significant shrinkage over time, but also show disagreements in results. These discrepancies may stem from differences in the method of brain assessment, a sample's age range, admixture of participants with vascular and metabolic risk factors, and selection of time windows for longitudinal follow-ups [1]. In healthy adults, significant shrinkage is observed in the cerebellum, neostriatum, posterior cingulate, and tertiary association cortices (dorsolateral orbital prefrontal, inferior parietal, and inferior temporal); lesser but still significant shrinkage is noted in the fusiform, insular, anterior cingulate, and parahippocampal cortices, whereas minimal changes or stability are found in the primary visual and somatosensory cortices [1]. Shrinkage of the medial temporal regions - the hippocampus and entorhinal cortex - has also been observed across multiple samples, with age-related acceleration observed in some [1]. Notably, the magnitude of regional shrinkage observed varies across studies, even when the samples are drawn from the same population, with some age trends less replicable than others, and significant individual variability in change found in virtually all regions. For example, substantial shrinkage of the dorsolateral prefrontal cortex and prefrontal white matter [13] was not observed in other samples studied in the same laboratory, whereas shrinkage of the cerebellum, the hippocampus, and the orbitofrontal cortex, accompanied by a relative stability of the primary visual cortex, was consistently replicated [13]. Thus, structural brain aging is clearly heterochronic;
50

Gerontology 2018;64:49-57

DOI: $10.1159 / 000479508$
Raz/Daugherty 
yet, the specific shape of the differential trajectories of change remains unknown.

In addition to volumetric findings, cortical thickness trajectories approximate the age-related reduction in regional volumes, with the cortical surface yielding lower estimates of age-related change [14]. The shape of agerelated change (linear, quadratic, or accelerated/decelerated exponential) is still unclear as very few studies included more than one follow-up assessment, thus hampering analysis of time-related trends. In thehippocampus, however, nonlinear shrinkage has been observed in several studies [e.g., 13, 15] that reported greater rates of change in older participants. Such acceleration may reflect a cumulative influence of age-related risk factors on the "normal" trajectories of aging and reinforce the notion of aging as a nonprogrammed process [2]. Shrinkage of the hippocampus, as well as of the prefrontal cortex, prefrontal white matter, and entorhinal cortex, has been linked to decline in cognitive performance or reduced ability to benefit from repeated testing $[16,17]$. The risk for vascular disease [13] and genetic variants associated with an increased risk for Alzheimer's disease and inflammation [18] exacerbate gray matter shrinkage.

The neurobiological and cytoarchitectonic foundations for differential gray matter shrinkage remain unclear. The neuropil - axons, dendrites, and their collateral branches - accounts for the bulk of gray matter and can expand and contract in response to multiple environmental changes [19], and this may be crucial to the gray matter shrinkage observed on MRI. Early-myelinating regions show fewer age-related differences and slower change [20]. Postmortem cortical myelin staining is related to age in an inverted- $U$ fashion; age-related differences in the heteromodal association cortices are greater than in the primary sensory regions [8], and the regions that myelinate earlier show lesser age-related shrinkage and smaller age-related differences [1]. Thus, loss of intralaminar myelin may contribute to age-related "sagging" of the cortical mantle, and investigation into agerelated changes in myelin content may be especially important for understanding brain aging.

\section{Age-Related Differences and Changes in White Matter Structure and Organization}

White matter is one of the main cerebral compartments devoid of neuron bodies, has a significant presence of astro- and oligodendroglia, contains less interstitial fluid than gray matter, and is relatively sparsely irrigated

Pathways to Brain Aging and Their Modifiers by isolated blood vessels. Most of the white matter axons are covered with myelin sheaths that enhance the speed and fidelity of neurotransmission and promote many energy, transport, and neurotrophic functions [21]. Myelin synthesis and maintenance of the myelin sheaths and the axonal microtubule transport system are energetically costly and depend on a steady blood flow. In this context, the relatively sparse vascularization of the densely packed white matter presents a maintenance problem, and the need for meeting substantial energetic demands makes the white matter particularly vulnerable to insults and dysfunctions that affect the energy supply to the brain.

Certain alterations to the white matter, such as white matter hyperintensities (WMH), are ubiquitous in older brains and can be observed on MRI. In addition to advanced age, WMH burden is associated with unfavorable cerebrovascular outcomes [22], metabolic dysfunction, and elevated neuroinflammation biomarker levels [23], as well as with poor cognitive performance [24]. WMH reflect multiple pathological changes that stem from subclinical ischemia and hypoperfusion, focal demyelination, and leakage of CSF and interstitial fluid through weakened blood-brain and CSF-brain barriers [22]. In healthy middle-aged and older persons, the WMH volume increases moderately over time, whereas genetic and physiological markers of vascular disease and inflammation are associated with an elevated WMH volume even in asymptomatic adults and may accelerate WMH progression [25]. Because WMH represent a late stage of pathological influences on normally appearing white matter (NAWM), their usefulness for revealing the mechanisms of nonpathological age-related cognitive changes is limited. A useful alternative is interrogating the white matter microstructure via observing and quantifying the diffusion behavior of water protons in the cerebral white matter regions that are characterized by various degrees of hindrance and barriers to diffusion, since it describes the state of NAWM before drastic changes become apparent.

The microstructural properties of the white matter are captured by diffusion tensor imaging (DTI) and can be summarized by several scalar indices computed from the eigenvalues of the diffusion tensor reflecting the anisotropy of water diffusion in tissue: fractional anisotropy (FA), mean diffusivity, axial diffusivity (AD), and radial diffusivity $(\mathrm{RD})$. Although they are sensitive to differences in white matter microstructural composition and fiber organization as well as multiple cellular and structural features $[26,27]$, DTI indices reflecting the anisotropy of water diffusion cannot provide specific information

Gerontology 2018;64:49-57

DOI: $10.1159 / 000479508$ 
about age-related changes in the white matter. Moreover, the influence of WMH that affect the diffusion characteristics in an age-dependent manner [28] is rarely considered in computing DTI indices. This adds further uncertainty to interpreting age differences in diffusion-related measures in the global white matter compartment that may include WMH versus NAWM.

Numerous DTI-based cross-sectional studies have revealed age-related differences in all DTI-derived indices across the preponderance of white matter tracts, with greater age differences in association tracks that connect the frontal and parietal heteromodal cortices than in projection fibers [29]. This pattern of age-related differences is in line with the first-in-last-out hypothesis [1]: regions of the brain that develop later are more vulnerable to ageassociated degeneration than those that evidence early growth.

Longitudinal investigations into white matter aging are still relatively rare, and most studies do not consider the influence of WMH on DTI-derived indices. The most consistent observation is a decline in FA across multiple white matter regions [e.g., 29-36]. Increases in AD and $\mathrm{RD}$ over time were reported in three studies [30-32]. In some samples, an advanced age at baseline predicted faster declines, thus suggesting a nonlinearity of the white matter aging trajectories [32]. Longitudinal studies of two independent samples of NAWM of healthy adults revealed declines in $\mathrm{AD}$ in association and projection fibers, and increases in $\mathrm{AD}$ in anterior commissural fibers over 2 years and up to 7 years, with FA and RD evidencing a less consistent pattern of change [32, 33]. Evaluations of individual differences in the age trajectories of change showed significant variability in one sample (a wide adult life span range [33]) but not in the other one (middle-age and older adults [34]). The white matter changes observed were aggravated by multiple vascular and metabolic [33] risk factors, as well as by genetic variants associated with an increased risk for Alzheimer's disease [35].

The impact of white matter changes on cognition is unclear, because of uncertainty about the neurobiological meaning of the DTI-derived indices and the dearth of longitudinal studies. In one sample, change in mean diffusivity but not FA was coupled with decline in working memory [37]. In very old persons, decline in perceptual speed was coupled with drop in FA [38], and in a large sample of septuagenarians, decline in FA was coupled with reduction in fluid intelligence scores [36]. However, in an adult life span sample, greater gains in associative memory were coupled with reduction in FA and increase in $\mathrm{RD}$ - i.e., changes in the direction that usually implies deterioration of the white matter [39]. Because the number of measurement occasions was limited to two, one can only state a temporal co-occurrence of two changes, without asserting a lead-lag relationship.

In sum, the results of longitudinal DTI studies suggest that early-myelinating regions are more resilient to the effects of aging than late-myelinating tracts such as the superior longitudinal fasciculus and the anterior limb of the internal capsule. Given the global nature of DTI metrics, their use cannot advance the understanding of white matter aging beyond a global statement of "being different," and the importance of developing more refined and specific models cannot be overstated.

New approaches have been proposed to account for the heterogeneity of the water diffusion determinants in the white matter by modeling multiple diffusion compartments (intracellular, extracellular, and intra-axonal water and CSF) and quantifying the distribution of axonal calibers as well as neurite density and orientation dispersion [40]. Too few studies have used these methods for assessing brain aging, and no longitudinal data are available as yet. Cross-sectional investigations into age differences in the white matter revealed contradicting results: widespread linear increases in neurite dispersion, without differences in fiber organization in the frontal regions [41]; or greater fiber orientation dispersion with age, but no age differences in neurite density [42]. In the largest cross-sectional study of white matter organization to date, a lower neurite density, lower tract complexity, and higher extracellular water diffusion were observed across most tracts in older participants [43]. It remains unclear, therefore, which organizational feature of the white matter is most sensitive to aging, and the lack of longitudinal studies compounds the uncertainty.

Because of myelin's importance for information processing and brain metabolism and the lack of specificity of DTI indices for myelin, estimating its content in vivo is a high priority. Several MRI methods allow evaluating the myelin content, albeit indirectly, via estimating the myelin water fraction (MWF) - a relative contribution of the magnitude of the short spin-spin $\mathrm{T}_{2}$ relaxation component $(10-40 \mathrm{~ms}$ at $3 \mathrm{~T})$ attributed to water molecules trapped between myelin sheaths to the total observed water signal. In addition, the intermediate $\mathrm{T}_{2}$ relaxation time $(60-75 \mathrm{~ms}$ at $3 \mathrm{~T})$ that corresponds to intra- and extracellular water reflects the axonal density and/or diameter [44]. To date, there have been no longitudinal studies of the MWF in normal aging. In one cross-sectional study, the myelin content in projection, commissural, and asso-
52

Gerontology 2018;64:49-57

DOI: $10.1159 / 000479508$
Raz/Daugherty 
ciation fiber regions evidenced an association with age that approximated an inverted-U shape, as would be expected from the pattern observed in postmortem studies [45]. In contrast, DTI-derived indices obtained from the same participants exhibited linear or null relationships to age, underscoring the lack of specificity of DTI for myelin. Another study of age differences in MWF [41] found no association between myelin content and age, but in that sample, only 3 participants were older than 60 years, and it is possible that the age range was too narrow to detect the lower myelin content in the older brains. Using a similar approach to characterizing water $\mathrm{T}_{2}$ relaxation (a multicomponent-driven equilibrium single pulse observation of $T_{1}$ and $T_{2}$ ), stronger associations between MWF and age in late-myelinating prefrontal subcortical white matter and the genu of the corpus callosum were observed in comparison to more precociously myelinated precentral and fusiform gyri [46]. A study using another proposed proxy for myelin content $\left(1 / \mathrm{T}_{1}\right.$ or $\mathrm{R}_{1}$ relaxation rate) reported curvilinear associations between age and $\mathrm{R}_{1}$ in a life span sample of healthy individuals with peaks of the age- $\mathrm{R}_{1}$ curves appearing earlier in the white matter tracts that correspond to sensory and motor regions than in the association fibers [47].

In sum, aging is associated with changes in white matter organization and microstructural properties. MRI proxies for myelin content (e.g., MWF and $\mathrm{R}_{1}$ ) show an inverted- $U$ association with age, similar to what is observed in postmortem studies, and regional differences suggest a greater age impact on the late-myelinating tracts. The lack of longitudinal studies and insufficient specificity of the commonly used methods hamper research on white matter aging.

\section{Brain Iron Homeostasis and Aging}

Several changes in metabolic function are considered to drive the progressive and cumulative changes in gray and white matter regions [1]. For example, aging is associated with a shift in homeostasis of bioactive metal ions - iron, copper, zinc, and manganese [7]. Postmortem studies show significantly higher amounts of iron in older brains $[48,49]$, and several studies have reported increased iron contents in neurodegenerative diseases that are associated with cognitive decline. Therefore, estimating the iron content in vivo can contribute to understanding structural brain aging.

Valid estimates of the regional iron content can be obtained via susceptibility-weighted imaging, a tech-

Pathways to Brain Aging and Their Modifiers nique that assesses local susceptibility effects produced by paramagnetic particles, including iron [50-52]. Cross-sectional in vivo study of healthy adults revealed elevated iron content in the striatum and several cortical areas of older adults compared to their younger counterparts [50]. A longitudinal increase in iron content within the striatum precedes and predicts regional shrinkage after 2 years [51] and up to 7 years in healthy adults [52].

Regional brain iron accumulation and the related shrinkage are associated with cognitive declines that are typical in aging and the progression of age-related neurodegenerative diseases [50]. In healthy adults, a longitudinal increase over 2 years in caudate iron content is coupled with lesser repeated-testing gains in verbal working memory [51], and an increase in hippocampal iron is associated with lesser gains in virtual Morris water maze navigation [17].

In sum, aging is accompanied by an increase in regional iron content that preferentially affects brain structures involved in motor control. Based on limited evidence, these increases precede regional shrinkage and are coupled with declines in cognitive and motor performance. The mechanisms of age-related iron accumulation remain unclear. It is plausible that the reductions in the brain's ability to manage iron sequestration and transport stem from a decrease in energy resources available for these functions. Thus, estimating age-related changes in brain energy metabolism is an important goal in the human neuroscience of aging.

\section{Iron and Brain Energy Metabolism}

The human brain is a unique organ that, despite of its relatively small size, draws a tenfold share of the body's energy and uses most of that energy for maintaining its information processing capabilities through electrical signaling, especially in glutamatergic neurons $[53,54]$. The main source of the brain's energy is adenosine triphosphate (ATP) synthesized in the mitochondria, with nutritional glucose and fatty acids, glycolysis, and $\beta$-oxidation of fatty acids, as well as a reduced form of nicotinamide adenine dinucleotide. ATP production depends on the mitochondrial membrane electron gradient, which is determined by electron transfer via a series of five iron-sulfur-rich protein megacomplexes located in the inner mitochondrial membrane [55]. Thus, iron is a critical player in energy metabolism and mitochondrial functions.

Gerontology 2018;64:49-57

DOI: $10.1159 / 000479508$ 
Iron's vital role in ATP synthesis is balanced by a significant potential for harm predicated on its propensity for generating, through a Fenton reaction, reactive oxygen species (ROS), also known as free radicals [49]. Free radicals that originate in the mitochondria and the endoplasmic reticulum as by-products of normal metabolic activity serve multiple useful functions, and low-level ROS-induced oxidative stress balanced by endogenous antioxidants may be necessary for normal cellular activity [49]. Excessive accumulation of ROS, however, is viewed as the fundamental phenomenon of the aging process, with related mitochondrial dysfunction as the centerpiece of the free-radical theory of aging [3]. ROS buildup upsets the equilibrium with antioxidants and produces oxidative stress that degrades mitochondrial membranes, impedes energy production in the mitochondria, promotes DNA mutations, and accelerates apoptosis [49]. The mitochondrial volume fraction, which is a proxy for cellular energy capacity, correlates with capillary density and is believed to correspond to energy use under the assumption that "what is not used tends to be pared away" [56]. Of note, mitochondria are more abundant in the neuropil than in neuronal bodies, and in the dendrites, mitochondrial density corresponds to demand on excitatory (glutamatergic) neurotransmission [57]. All these vital functions depend on the availability of iron and are vulnerable to iron-related oxidative stress.

In normally functioning cells, iron transported into the mitochondria is tightly controlled. Rendered harmless by binding proteins, iron is released only upon a signal from mitochondria for immediate metabolism [49]. With advancing age, the sequestration processes begin to falter and iron homeostasis shifts to a dangerous level of free, unbound iron [50]. By producing ROS and thus enabling oxidative stress, free iron exacerbates mitochondrial dysfunction, which interferes with the synthesis and availability of high-energy phosphates in the cortical neuropil, subcortical structures, and white matter, and thus promotes age-related tissue degeneration [49]. The disproportionate depletion of energy resources affects processes such as the synthesis and maintenance of myelin, as well as the uptake and sequestration of iron released by myelin decay [58]. In addition to rendering intercellular signaling less efficient and reliable, declines in the availability of high-energy phosphates impede the maintenance and turnover of membrane phospholipids, which are necessary for synaptic plasticity, branching of dendrites and axon terminals, and formation of astrocytes [7]. Thus, the iron content assessed in conjunction with energy metabolism, the myelin content, and the neuropil volume can provide a window into understanding the mechanisms of brain aging. In that context, in vivo assessment of brain energy metabolism is of particular importance.

\section{Brain Aging and Energy Metabolism}

To meet its high energetic demands, the adult brain produces and consumes almost $6 \mathrm{~kg}$ of ATP per day. Another high-energy phosphate, phosphocreatine (PCr), serves as a reservoir for the rapid generation of ATP via creatine kinase reaction, a pathway that is approximately 5-6 times more active than ATP production via the mitochondrial ATPase pathway [59]. Creatine kinase is also involved in transferring PCr out of mitochondria. Therefore, the PCr metabolic level assessed in vivo is a direct reflection of the high-energy storage capacity and energy demand. These metabolites can be estimated in vivo by phosphorus magnetic resonance spectroscopy $\left({ }^{31} \mathrm{P}\right.$ MRS), which has the major advantage of directly measuring inorganic phosphate (PCr) and ATP as well as phosphomonoesters and the phosphodiesters glycerophosphocholine and glycerophosphoethanolamine (GPC+GPE) in localized brain areas [60]. This technique allows assessment of the precursors and breakdown products of membrane phospholipids, and thereby a means of evaluating the extent of neuropil expansion and contraction [60].

Extant ${ }^{31} \mathrm{P}$ MRS studies of aging are rare and are exclusively cross-sectional. Preliminary findings indicate, nevertheless, significant age-related differences in several key indicators of neural membrane turnover and mitochondrial function. In older adults, the phosphatidylcholine plus phosphatidylethanolamine (PC+PE)/GPC+GPE ratio was greater in older adults [61], whereas the intracellular $\mathrm{pH}$ was lower, and $\mathrm{PCr}$ was higher, in middle-aged than in younger subjects $[62,63]$, which suggests a less efficient energy metabolism with advanced age. In aging rats, age-related elevations of $\mathrm{PCr}$ and $\mathrm{PC}+\mathrm{PE}$ were mirrored by in vitro reduction in ATP, as well as by a peripheral decrease in antioxidant catalase [63]. Higher wholebrain gray matter PCr levels nonetheless have been linked to better performance on an age-sensitive response inhibition task in healthy older adults [64], suggesting that its age-related elevation may be compensatory. In sum, despite being still quite sparse, the extant literature on ${ }^{31} \mathrm{P}$ MRS in normal aging suggests that it may be a unique tool for exploring brain energy metabolism in vivo.
Raz/Daugherty 


\section{FRIENDS Model}

Six decades ago, Harman [3] proposed that aging could be an expression of cumulative damage inflicted on the cellular machinery by ROS, also known as free radicals. Although some of his tenets have been questioned, the free-radical theory of aging remains arguably the most influential conceptual framework in biogerontology. The FRIENDS model of human aging proposed here was inspired by Harman's conceptualization of aging and its modern elaborations [65], as well as by the disposable soma theory (DST) [2]. The core idea of the DST is that life span development depends on the optimal allocation of metabolic resources with limited investment in maintenance and repair. It views aging not as a programmed biological process unfolding on a predetermined schedule, but as a phenomenon marked by a progressively increasing probability of systemic failure, which may reflect built-in imperfections of cellular metabolic processes [66]. The central corollary of the DST is therefore the attribution of age-related declines in brain functions and in their cognitive expression to a gradual reduction in energy resources available for the maintenance of critically important brain infrastructures.

The FRIENDS model (Fig. 1) assumes that the core cause of cognitive aging is a generalized and chronic decline in the availability of energy resources. This gradual energy deprivation is focused in the cell power house: the mitochondria. In the brain, hypoenergetic mitochondria underlie impairment of multiple energy-dependent cellular processes, including the sequestration and transport of iron, which in turn causes a reduction in mitochondrial energy metabolism. This positive-feedback cascade leads to further mitochondrial dysfunction: the subsequent release of free iron leads to a shift in ROS homeostasis, and excess ROS further damage the mitochondria. The amount of energy available for energetically costly processes such as the maintenance of cell membranes and myelin, homeostasis of important ions (e.g., calcium), and preservation of neurotransmission is gradually reduced. The late-myelinating tracts that require greater myelin maintenance into middle age (i.e., connection between association areas) are expected to be disproportionally affected by this energy crisis. Endothelial dysfunction accompanying normal aging [4] makes blood vessels vulnerable to loss of function, thereby further exacerbating the energy crisis by reducing the availability of essential substrates to the brain. Thus, one can expect greater age-related changes in the regions at the periphery of the brain's vascular supply system, e.g., the dorsolat-

Pathways to Brain Aging and Their Modifiers

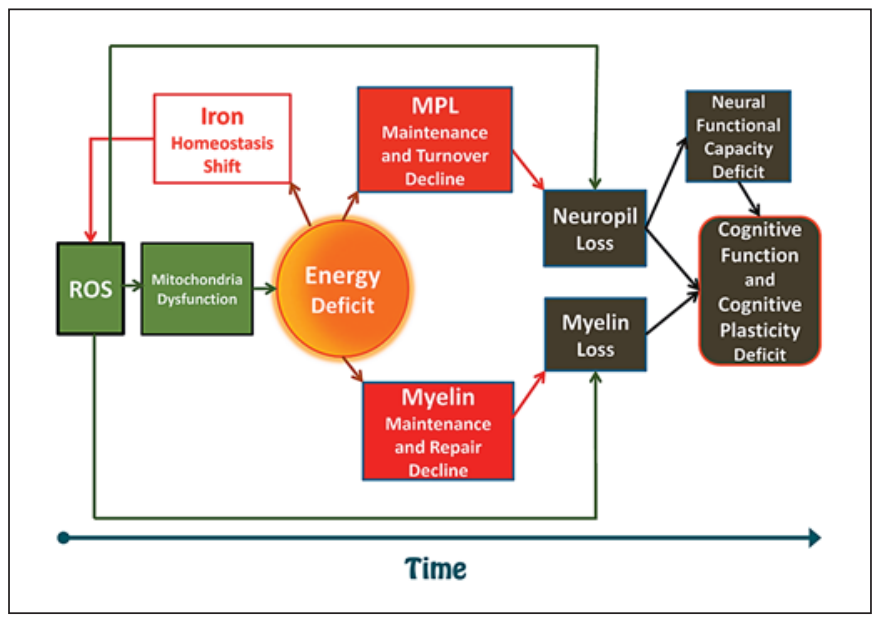

Fig. 1. FRIENDS model of cognitive aging. A gradually reduced production of energy sources (ATP and NADH) in the brain leads to an impairment of the sequestration and transport of iron, which in turn causes a reduction in mitochondrial energy metabolism. Mitochondrial dysfunction leads to a shift in reactive oxygen species (ROS) homeostasis, and excess of ROS further damages the mitochondria. As a result of this cascade, the energy available for the maintenance of cell membranes and myelin is further reduced and brain plasticity is abridged. Myelin and the neuropil are gradually reduced, and with that loss, the information processing capabilities of the brain networks are degraded, with the system becoming progressively noisier. Noise in the information processing system affects all cognitive operations, with the more complex of them fairing worst. MPL, membrane phospholipid.

eral prefrontal and inferior parietal cortices. These deficits further hamper brain plasticity and reduce its systemic capacity to compensate for the losses of critical elements and connections in its information processing circuits. With degradation of the ability to form new connections and maintain the existing circuitry, the brain becomes a progressively noisier system. Noise in the information processing system affects all cognitive operations, with the more complex and least automated of them fairing worst (hence, differential aging of fluid vs. crystallized intelligence).

Although many of the steps proposed in the FRIENDS model have been established in animal models, its testing in humans presents multiple challenges. Some elements of FRIENDS, however, have been already verified in human studies; others can be assessed by the existing technical means reviewed here. The key challenge to testing the model is the lack of noninvasive, in vivo ways of verifying its core tenet: the age-related increase in ROS as the enabler and accelerator of mitochondrial dysfunction. We propose that assessing the brain's regional nonheme iron

Gerontology 2018;64:49-57 DOI: $10.1159 / 000479508$ 
content - a primary generator of ROS - via MRI can be a useful proxy for gauging oxidative stress [50]. Although iron tends to preferentially accumulate in the brain structures associated with motor control, it is likely to be a global marker of impaired ROS homeostasis and a sign of global energy deficit. In vulnerable structures that are more dependent than others on energy resources, such global deficits can be expressed as a local change observed in a differential aging pattern. As reviewed, several extant methods for assessing brain structure integrity, energy production, blood flow, and vascular and metabolic dysfunction can be harnessed for appraising the aging system. To move beyond descriptive statements of age differences, multimodal assessment of the aging brain in multi-occasion longitudinal study is needed. Such assess- ment will not only elucidate the temporal dynamics of specific brain properties but will also test the hypotheses about the temporal order of changes unfolding during the life span as outlined in the FRIENDS model.

\section{Acknowledgements}

The work on this review was supported by a grant (R01AG011230) from the National Institute on Aging to N.R. and a Beckman Institute Postdoctoral Fellowship (University of Illinois at Urbana-Champaign), with funding provided by the Arnold and Mabel Beckman Foundation, to A.M.D. We gratefully acknowledge the constructive comments by Jeffrey A. Stanley and the anonymous reviewers.

\section{References}

1 Kennedy KM, Raz N: Normal aging of the brain; in Toga AW (ed): Brain Mapping: An Encyclopedia Reference. London/San Diego/ Waltham/Oxford, Elsevier, 2015, vol 3, pp 603-617.

2 Kirkwood TB, Melov S: On the programmed/ non-programmed nature of ageing within the life history. Curr Biol 2011;21:R701-R707.

3 Harman D: Aging: a theory based on free radical and radiation chemistry. J Gerontol 1956; 11:298-300.

4 Singer J, Trollor JN, Baune BT, Sachdev PS, Smith E: Arterial stiffness, the brain and cognition: a systematic review. Ageing Res Rev 2014;15:16-27.

5 Bartzokis G: Age-related myelin breakdown: a developmental model of cognitive decline and Alzheimer's disease. Neurobiol Aging 2004;25:5-18.

6 Kemper TL: Neuroanatomical and neuropathological changes during aging and in dementia; in Albert ML, Knoepfel EJE (eds): Clinical Neurology of Aging. New York, Oxford University Press, 1994, pp 3-67.

7 Kaes T: Die Grosshirnrinde des Menschen in ihren Massen und in ihrem Fasergehalt. Jena, Gustav Fisher, 1907.

8 Wang $\mathrm{P}$, Wang ZY: Metal ions influx is a double edged sword for the pathogenesis of Alzheimer's disease. Ageing Res Rev 2017;35: 265-290.

9 Ghisletta P, Aichele S: Quantitative methods in psychological aging research: a mini-review. Gerontology 2017, Epub ahead of print.

10 Molenaar PCM: A manifesto on psychology as idiographic science: bringing the person back into scientific psychology, this time forever. Measurement 2004;2:201-218.

11 Maxwell SE, Cole DA: Bias in cross-sectional analyses of longitudinal mediation. Psychol Methods 2007;12:23-44.
12 Lindenberger U, von Oertzen T, Ghisletta P, Hertzog C: Cross-sectional age variance extraction: what's change got to do with it? Psychol Aging 2011;26:34-47.

13 Raz N, Lindenberger U, Rodrigue KM, Kennedy KM, Head D, Williamson A, Dahle C, Gerstorf D, Acker JD: Regional brain changes in aging healthy adults: general trends, individual differences, and modifiers. Cereb Cortex 2005;15:1676-1689.

14 Storsve AB, Fjell AM, Tamnes CK, Westlye LT, Overbye K, Aasland HW, Walhovd KB: Differential longitudinal changes in cortical thickness, surface area and volume across the adult life span: regions of accelerating and decelerating change. J Neurosci 2014;34:84888498.

15 Crivello F, Tzourio-Mazoyer N, Tzourio C, Mazoyer B: Longitudinal assessment of global and regional rate of grey matter atrophy in 1,172 healthy older adults: modulation by sex and age. PLoS One 2014;9:e114478.

16 Kramer JH, Mungas D, Reed BR, Wetzel ME, Burnett MM, Miller BL, Weiner MW, Chui HC: Longitudinal MRI and cognitive change in healthy elderly. Neuropsychology 2007;21: 412-418.

17 Daugherty AM, Raz N: A virtual water maze revisited: two-year changes in navigation performance and their neural correlates in healthy adults. Neuroimage 2017;146:492506.

18 Harrison TM, Mahmood Z, Lau EP, Karacozoff AM, Burggren AC, Small GW, Bookheimer SY: An Alzheimer's disease genetic risk score predicts longitudinal thinning of hippocampal complex subregions in healthy older adults. eNeuro 2016;3:ENEURO. 0098-16.2016.
19 Kassem MS, Lagopoulos J, Stait-Gardner T, Price WS, Chohan TW, Arnold JC, Hatton SN, Bennett MR: Stress-induced grey matter loss determined by MRI is primarily due to loss of dendrites and their synapses. Mol Neurobiol 2013;47:645-661.

20 Raz N: Ageing and the brain; in Encyclopedia of Life Sciences. London, Macmillan Reference Ltd, 2001.

21 Beaulieu C: The biological basis of diffusion anisotropy; in Johansen-Berg H, Behrens TEJ (eds): Diffusion MRI: From Quantitative Measurement to in vivo Neuroanatomy, ed 2. San Diego, Elsevier, 2014, pp 155-185.

22 Pantoni L, Garcia JH: Pathogenesis of leukoaraiosis: a review. Stroke 1997;28:652-659.

23 Portet F, Brickman AM, Stern Y, Scarmeas N, Muraskin J, Provenzano FA, Berr C, Bonafé A, Artero S, Ritchie K, Akbaraly TN: Metabolic syndrome and localization of white matter hyperintensities in the elderly population. Alzheimers Dement 2012;8(suppl): S88-S95.e1.

24 Gunning-Dixon FM, Raz N: The cognitive correlates of white matter abnormalities in normal aging: a quantitative review. Neuropsychology 2000;14:224-232.

25 Sabayan B, van der Grond J, Westendorp RG, van Buchem MA, de Craen AJ: Accelerated progression of white matter hyperintensities and subsequent risk of mortality: a 12-year follow-up study. Neurobiol Aging 2015;36: 2130-2135.

26 Jones DK, Knösche TR, Turner R: White matter integrity, fiber count, and other fallacies: the do's and don'ts of diffusion MRI. Neuroimage 2013;73:239-254.

27 Jeurissen B, Leemans A, Tournier JD, Jones $\mathrm{DK}$, Sijbers J: Investigating the prevalence of complex fiber configurations in white matter tissue with diffusion magnetic resonance imaging. Hum Brain Mapp 2013;34:2747-2766. 
28 Vernooij MW, de Groot M, van der Lugt A, Ikram MA, Krestin GP, Hofman A, Niessen WJ, Breteler MM: White matter atrophy and lesion formation explain the loss of structural integrity of white matter in aging. Neuroimage 2008;43:470-477.

29 Salat DH: Diffusion tensor imaging in the study of aging and age-associated neural disease; in Johansen-Berg H, Behrens TEJ (eds): Diffusion MRI: From Quantitative Measurement to in vivo Neuroanatomy, ed 2. San Diego, Elsevier, 2014, pp 257-281.

30 Barrick TR, Charlton RA, Clark CA, Markus HS: White matter structural decline in normal ageing: a prospective longitudinal study using tract-based spatial statistics. Neuroimage 2010;51:565-577.

31 Sexton CE, Storsve AB, Walhovd KB, Johansen-Berg H, Fjell AM: Poor sleep quality is associated with increased cortical atrophy in community-dwelling adults. Neurology 2014; 83:967-973.

32 Storsve AB, Fjell AM, Yendiki A, Walhovd KB: Longitudinal changes in white matter tract integrity across the adult lifespan and its relation to cortical thinning. PLoS One 2016; 11:e0156770.

33 Bender AR, Raz N: Normal-appearing cerebral white matter in healthy adults: mean change over 2 years and individual differences in change. Neurobiol Aging 2015;36:18341848.

34 Bender AR, Völkle MC, Raz N: Differential aging of cerebral white matter in middle-aged and older adults: a seven-year follow-up. Neuroimage 2016;125:74-83.

35 Riekmann A, Van Dijk KRA, Sperling RA, Johnson KA, Buckner RL, Hedden T: Accelerated decline in white matter integrity in clinically normal individuals at risk for Alzheimer's disease. Neurobiol Aging 2016;42:177188.

36 Ritchie SJ, Bastin ME, Tucker-Drob EM, Maniega SM, Engelhardt LE, Cox SR, Royle NA, Gow AJ, Corley J, Pattie A, Taylor AM, Valdés Hernández Mdel C, Starr JM, Wardlaw JM, Deary IJ: Coupled changes in brain white matter microstructure and fluid intelligence in later life. J Neurosci 2015;35:86728582.

37 Charlton RA, Schiavone F, Barrick TR, Morris RG, Markus HS: Diffusion tensor imaging detects age related white matter change over a 2 year follow-up which is associated with working memory decline. J Neurol Neurosurg Psychiatry 2010;81:13-19.

38 Lövdén M, Köhncke Y, Laukka EJ, Kalpouzos G, Salami A, Li TQ, Fratiglioni L, Bäckman L: Changes in perceptual speed and white matter microstructure in the corticospinal tract are associated in very old age. Neuroimage 2014; 102(pt 2):520-530.

39 Bender AR, Prindle JJ, Brandmaier AM, Raz $\mathrm{N}$ : White matter and memory in healthy adults: coupled changes over two years. Neuroimage 2016;131:193-204.
40 Assaf Y, Blumenfeld-Katzir T, Yovel Y, Basser PJ: AxCaliber: a method for measuring axon diameter distribution from diffusion MRI. Magn Reson Med 2008;59:1347-1354.

41 Billiet T, Vandenbulcke M, Mädler B, Peeters R, Dhollander T, Zhang H, Deprez S, Van den Bergh BR, Sunaert S, Emsell L: Age-related microstructural differences quantified using myelin water imaging and advanced diffusion MRI. Neurobiol Aging 2015;36:2107-2121.

42 Kodiweera C, Alexander AL, Harezlak J, McAllister T, Wu YC: Age effects and sex differences in human brain white matter of young to middle-aged adults: a DTI, NODDI, and q-space study. Neuroimage 2016;128: 180-192.

43 Cox SR, Ritchie SJ, Tucker-Drob EM, Liewald DC, Hagenaars SP, Davies G, Wardlaw JM, Gale CR, Bastin ME, Deary IJ: Ageing and brain white matter structure in 3,513 UK Biobank participants. Nat Commun 2016;7: 13629.

44 Arshad M, Stanley JA, Raz N: Test-retest reliability and concurrent validity of in vivo myelin content indices: myelin water fraction and calibrated $\mathrm{T}_{1} \mathrm{w} / \mathrm{T}_{2} \mathrm{w}$ image ratio. Hum Brain Mapp 2017;38:1780-1790.

45 Arshad M, Stanley JA, Raz N: Adult age differences in subcortical myelin content are consistent with protracted myelination and unrelated to diffusion tensor imaging indices. Neuroimage 2016;143:26-39.

46 Dean DC 3rd, Hurley SA, Kecskemeti SR, O'Grady JP, Canda C, Davenport-Sis NJ, Carlsson CM, Zetterberg H, Blennow K, Asthana S, Sager MA, Johnson SC, Alexander AL, Bendlin BB: Association of amyloid pathology with myelin alteration in preclinical Alzheimer Disease. JAMA Neurol 2017;74: 41-49.

47 Yeatman JD, Wandell BA, Mezer AA: Lifespan maturation and degeneration of human brain white matter. Nat Commun 2014;5: 4932.

48 Hallgren B, Sourander P: The effect of age on the non-haemin iron in the human brain. J Neurochem 1958;3:41-51.

49 Ward RJ, Zucca FA, Duyn JH, Crichton RR, Zecca L: The role of iron in brain ageing and neurodegenerative disorders. Lancet Neurol 2014;13:1045-1060.

50 Daugherty A, Raz N: Appraising the role of iron in brain aging and cognition: promises and limitations of MRI methods. Neuropsychol Rev 2015;25:272-287.

51 Daugherty AM, Haacke EM, Raz N: Increase in iron content predicts shrinkage of the striatum and changes in verbal working memory in healthy adults. J Neurosci 2015;35:67316743.
52 Daugherty AM, Raz N: Accumulation of iron in the putamen predicts its shrinkage in healthy older adults: a multi-occasion longitudinal study. Neuroimage 2016;128:11-20.

53 Attwell D, Laughlin SB: An energy budget for signaling in the grey matter of the brain. J Cereb Blood Flow Metab 2001;21:1133-1145.

54 Howarth C, Gleeson P, Attwell D: Updated energy budgets for neural computation in the neocortex and cerebellum. J Cereb Blood Flow Metab 2012;32:1222-1232.

55 Dromparis P, Michelakis ED: Mitochondria in vascular health and disease. Annu Rev Physiol 2013;75:95-126.

56 Perge JA, Niven JE, Mugnaini E, Balasubramanian V, Sterling P: Why do axons differ in caliber? J Neurosci 2012;32:626-638.

57 Kageyama GH, Wong-Riley MT: The histochemical localization of cytochrome oxidase in the retina and lateral geniculate nucleus of the ferret, cat, and monkey, with particular reference to retinal mosaics and ON/OFFcenter visual channels. J Neuroscience 1984;4: 2445-2459.

58 Haider L: Inflammation, iron, energy failure, and oxidative stress in the pathogenesis of multiple sclerosis. Oxid Med Cell Longev 2015;2015:725370.

59 Schlattner U, Tokarska-Schlattner M, Wallimann T: Mitochondrial creatine kinase in human health and disease. Biochim Biophys Acta 2006; 1762:164-180.

60 Pettegrew JW, Klunk WE, Panchalingam K, McClure RJ, Stanley JA: Molecular insights into neurodevelopmental and neurodegenerative diseases. Brain Res Bull 2000;53:455469.

61 Wijnen JP, Scheenen TW, Klomp DW, Heerschap A: ${ }^{31} \mathrm{P}$ magnetic resonance spectroscopic imaging with polarisation transfer of phosphomono- and diesters at $3 \mathrm{~T}$ in the human brain: relation with age and spatial differences. NMR Biomed 2010;23:968-976.

62 Forester BP, Berlow YA, Harper DG, Jensen JE, Lange N, Froimowitz MP, Ravichandran C, Iosifescu DV, Lukas SE, Renshaw PF, Cohen BM: Age-related changes in brain energetics and phospholipid metabolism. NMR Biomed 2010;23:242-250.

63 Vančová O, Bačiak L, Kašparová S, Kucharská J, Palacios HH, Horecký J, Aliev G: In vivo and in vitro assessment of brain bioenergetics in aging rats. J Cell Mol Med 2010;14:26672674 .

64 Harper DG, Joe EB, Jensen JE, Ravichandran C, Forester BP: Brain levels of high-energy phosphate metabolites and executive function in geriatric depression. Int J Geriatr Psychiatry 2016;31:1241-1249.

65 Sohal RS, Orr WC: The redox stress hypothesis of aging. Free Radic Biol Med 2012;52: 539-555.

66 Gladyshev VN: Origin of aging: imperfectness-driven non-random damage defines the aging process and control of lifespan. Trends Genet 2013;29:506-512. 